\title{
Uso de aminoetoxivinilglicina na maturação e queda pré-colheita de maçãs 'Imperial Gala'
}

\author{
Fernando José Hawerroth ${ }^{1}$, José Luiz Petri², Gabriel Berenhauser Leite ${ }^{3}$, Marcelo Couto ${ }^{3}$
}

\begin{abstract}
RESUMO
O uso de técnicas de controle da maturação dos frutos é fundamental na produção de macieiras 'Gala', no intuito de aumentar a capacidade de conservação dos frutos e diminuir a queda de frutos em pré-colheita. Objetivou-se, com este trabalho, avaliar o efeito de diferentes doses, épocas de aplicação e parcelamento de aplicações de aminoetoxivinilglicina (AVG), sobre a maturação e queda pré-colheita de maçãs 'Imperial Gala'. O experimento foi conduzido em pomar comercial, no município de Fraiburgo/SC. Utilizando-se o delineamento em blocos casualizados, com cinco repetições, compostas por uma planta cada, foram avaliados os seguintes tratamentos: 1. testemunha (sem aplicação); $2.120 \mathrm{~g} \mathrm{ha}^{-1} \mathrm{de}$ AVG, aplicados 4 semanas antes do ponto de colheita presumido (SAPC); 3. $60 \mathrm{~g}$ ha-1 de AVG (4 SAPC) + $60 \mathrm{~g} \mathrm{ha}^{-1}$ de AVG (2 SAPC); $4.30 \mathrm{~g} \mathrm{ha}^{-1}$ de AVG (2 SAPC) + $30 \mathrm{~g} \mathrm{ha}^{-1}$ de AVG no ponto de colheita (PC); 5.90 $\mathrm{g} \mathrm{ha}^{-1}$ de AVG (2 SAPC); $6.30 \mathrm{~g} \mathrm{ha}^{-1} \mathrm{de}$ AVG (1 SAPC) + $30 \mathrm{~g} \mathrm{ha}^{-1}$ de AVG (ponto de colheita - PC) + $30 \mathrm{~g} \mathrm{ha}^{-1} \mathrm{de} \mathrm{AVG}(7$ dias após o ponto de colheita - DAPC). O uso de AVG, independentemente da época, concentração e parcelamento das aplicações, reduziu a queda pré-colheita de maçãs 'Imperial Gala'. O grau de amarelamento da epiderme dos frutos mostra-se menor em frutos tratados com AVG. O atraso no início da colheita, proporcionado pelo AVG, determinou aumento da massa média dos frutos e os melhores resultados foram obtidos com a aplicação de $30 \mathrm{~g} \mathrm{ha}^{-1}$ de $\mathrm{AVG}(2$ $\mathrm{SAPC})+30 \mathrm{~g} \mathrm{ha}^{-1} \mathrm{de}$ AVG (PC) $+30 \mathrm{~g} \mathrm{ha}^{-1} \mathrm{de}$ AVG (7 DAPC).
\end{abstract}

Palavras-chave: Malus domestica, AVG, qualidade de frutos, abscisão de frutos, manejo da colheita.

\section{ABSTRACT}

\section{Aminoethoxyvinylglycine on maturation and preharvest drop control of 'Imperial Gala' apples}

The use of techniques for controlling fruit maturation is critical in the production of 'Gala' apples in order to increase postharvest fruit preservation and reduce preharvest fruit drop. The objective of this work was to evaluate the effect of different concentrations, application times and multiple applications of aminoethoxyvinylglycine (AVG) on maturation and preharvest fruit drop of 'Imperial Gala' apples. The experiment was carried out in a commercial orchard in the municipality of Fraiburgo, SC, Brazil. The experiment was arranged in a completely randomized block design, with five replications of individual trees. The following treatments were evaluated: 1 . control (no application); $2.120 \mathrm{~g} \mathrm{ha}^{-1}$ of AVG sprayed four weeks before the predicted harvest date (WBPH); $3.60 \mathrm{~g} \mathrm{ha}^{-1}$ of AVG (4 WBPH) $+60 \mathrm{~g} \mathrm{ha}^{-1}$ of AVG (2 WBPH); $4.30 \mathrm{~g} \mathrm{ha}^{-1}$ of AVG (2 WBPH) $+30 \mathrm{~g} \mathrm{ha}^{-1}$ of AVG at predict harvest (PH); $5.90 \mathrm{~g} \mathrm{ha}^{-1}$ of AVG (2 WBPH); e 6. $30 \mathrm{~g} \mathrm{ha}^{-1}$ of AVG $(1 \mathrm{WBPH})+30 \mathrm{~g} \mathrm{ha}^{-1}$ of AVG (predict harvest - PH) $+30 \mathrm{~g} \mathrm{ha}^{-1}$ of AVG (seven days after predict harvest - DAPH). Regardless of application time, concentration, single or multiple spray applications, the use of AVG reduced the preharvest fruit drop of 'Imperial Gala' apples. The color change on fruit surface was lower in fruits treated with AVG. The harvest delay caused by AVG treatments increased the mean fruit weight, and best results were obtained with application of $30 \mathrm{~g} \mathrm{ha}^{-1}$ of AVG $(1 \mathrm{WBPH})+30 \mathrm{~g} \mathrm{ha}^{-1}$ of AVG $(\mathrm{PH})+30 \mathrm{~g} \mathrm{ha}^{-1}$ of AVG (7 DAPH).

Key words: Aminoethoxyvinylglycine, fruit abscission, fruit quality, harvest management, Malus domestica Borkhausen.

\footnotetext{
Recebido para publicação em 20/01/2011 e aprovado em 29/09/2011

'Engenheiro-Agrônomo, Doutor. Embrapa Agroindústria Tropical, Rua Doutora Sara Mesquita, 2270, Planalto do Pici, 60511-110, Fortaleza, Ceará, Brasil. fernando@cnpat.embrapa.br;

${ }^{2}$ Engenheiro-Agrônomo, Mestre. Epagri - Estação Experimental de Caçador, Rua Abílio Franco, 1500, Bom Sucesso, 89500-000, Caçador, Santa Catarina, Brasil. petri@epagri.sc.gov.br; ${ }^{3}$ Engenheiro-Agrônomo, Doutor. Epagri - Estação Experimental de Caçador, Rua Abílio Franco, 1500, Bom Sucesso, 89500-000, Caçador, Santa Catarina, Brasil. gabriel@epagri.sc.gov.br, marcelocouto@epagri.sc.gov.br.
}

Rev. Ceres, Viçosa, v. 58, n.5, p. 612-618, set/out, 2011 


\section{INTRODUÇÃO}

O cultivar Gala e seus clones representam, aproximadamente, $60 \%$ da produção brasileira de maçãs, estimada pela Faostat (2010) em 1,22 milhões de toneladas, no ciclo 2008/2009. Este cultivar apresenta rápida maturação no período de colheita (Argenta, 1992), resultante de sua alta sensibilidade e alta taxa de produção autocatalítica de etileno $\left(\mathrm{C}_{2} \mathrm{H}_{4}\right)$ (Brackmann, 1992). Graças ao grande rendimento e à grande área de exploração do cultivar, parte da produção de maçãs Gala é, inevitavelmente, colhida após a maturação ideal para armazenamento em longo prazo, determinando curta vida pós-colheita dos frutos (Argenta et al., 2006). Além disso, são observadas perdas significativas da produção, em consequência da alta sensibilidade do cultivar Gala à queda de frutas em pré-colheita (Petri et al., 2007).

Considerando-se que macieiras 'Gala' apresentam maturação acelerada e são propensas à queda de frutos na pré-colheita, o manejo da maturação dos frutos apresenta grande importância econômica, nas condições brasileiras. Segundo Steffens et al. (2008), a utilização de reguladores de crescimento pode retardar a maturação e a colheita de frutos, possibilitando racionalizar o uso de mão de obra na colheita e reduzir as perdas durante o armazenamento. A aplicação de aminoetoxivinilglicina (AVG), substância inibidora da biossíntese do etileno (Greene, 2005), mostra-se eficiente no controle da maturação de frutos e no controle da queda de maçãs 'Gala' em pré-colheita (Amarante et al., 2002; Greene, 2005; Steffens et al., 2005; Petri et al. 2007).

Comercialmente, a AVG é utilizada para controle da queda de frutos em pré-colheita e retardamento da maturação dos frutos, sendo aplicada quatro semanas antes do ponto de colheita (Yuan \& Carbaugh, 2007). No entanto, a aplicação de AVG, na época recomendada, pode reduzir o acúmulo de pigmentos antociânicos e diminuir a degradação da clorofila na epiderme dos frutos, determinando atraso no desenvolvimento da coloração vermelha das maçãs, segundo Wang \& Dilley (2001), Amarante $e t$ al. (2002), Steffens et al. (2005) e Lurie (2008). Além disso, os índices de firmeza de polpa, acidez, degradação de amido e cor de película dos frutos mostram-se variáveis com a aplicação de AVG (Clayton et al., 2000), sendo as respostas variáveis com as concentrações, as épocas de aplicação e os cultivares utilizados (Greene, 2005; Petri et al., 2007). Por essa razão, torna-se importante a avaliação dos efeitos de diferentes épocas e de concentrações de AVG, assim como do parcelamento de aplicações, a fim de aumentar a eficiência deste fitorregulador no controle da maturação e da queda pré-colheita de frutos, sem comprometer o desenvolvimento da coloração dos frutos. Assim, objetivou-se, com o presente trabalho, avaliar o efeito de diferentes doses, épocas de aplicação e parcelamento de aplicações de AVG, sobre a maturação e queda pré-colheita de maçãs 'Imperial Gala'.

\section{MATERIAL E MÉTODOS}

O experimento foi realizado em pomar comercial, no município de Fraiburgo/SC ( $26^{\circ} 46^{\prime} \mathrm{S}, 51^{\circ} \mathrm{W}$, altitude 960 metros), durante o ciclo 2006/2007. Foram utilizadas macieiras 'Imperial Gala', enxertadas sobre o porta-enxerto M26, com densidade de plantio de 2500 plantas ha ${ }^{-1}$, com espaçamento de $4 \mathrm{~m}$ entre linhas e $1 \mathrm{~m}$ entre plantas, sendo as plantas conduzidas no sistema em líder central e manejadas de acordo com recomendações do sistema de produção da macieira (Sanhueza et al., 2006).

Foram testados os seguintes tratamentos: 1. testemunha (sem aplicação); 2. $120 \mathrm{~g} \mathrm{ha}^{-1}$ de AVG, aplicados 4 semanas antes do ponto de colheita presumido (SAPC); 3. $60 \mathrm{~g} \mathrm{ha}^{-1} \mathrm{de}$ AVG (4 SAPC) + $60 \mathrm{~g} \mathrm{ha}^{-1}$ de AVG (2SAPC); $4.30 \mathrm{~g} \mathrm{ha}^{-1}$ de AVG (2 SAPC) $+30 \mathrm{~g} \mathrm{ha}^{-1}$ de AVG, no ponto de colheita (PC); $5.90 \mathrm{~g} \mathrm{ha}^{-1}$ de AVG (2 SAPC); e $6.30 \mathrm{~g} \mathrm{ha}^{-}$ ${ }^{1} \mathrm{de}$ AVG (1 SAPC) + $30 \mathrm{~g} \mathrm{ha}^{-1} \mathrm{de}$ AVG (PC) $+30 \mathrm{~g} \mathrm{ha}^{-1} \mathrm{de}$ AVG, aplicados 7 dias após ponto de colheita (DAPC). Como fonte de AVG foi utilizado o produto comercial Retain $^{\circledR}$, contendo $15 \%$ do ingrediente ativo. Os tratamentos foram aplicados com pulverizador costal motorizado, com bico leque D-S, utilizando-se um volume médio de calda de $1000 \mathrm{~L} \mathrm{ha}^{-1}$, sendo adicionado o espalhante adesivo siliconado Silwet ${ }^{\circledR}$, na concentração de $0,05 \%$, nos tratamentos com AVG.

No início da maturação dos frutos do tratamento testemunha, foram realizadas amostragens de frutos, para a determinação dos parâmetros relacionados com a sua maturação. As amostras foram compostas de cinco frutos por repetição, colhidos aleatoriamente no terço médio das plantas. Foram efetuadas as análises da firmeza de polpa, sólidos solúveis, índice de iodo-amido, cor de fundo da epiderme e concentração de etileno dos frutos. A firmeza de polpa foi quantificada em duas extremidades opostas, da superfície do fruto, na sua porção equatorial, utilizando-se penetrômetro eletrônico com ponteira de $11 \mathrm{~mm}$ (Güss). A secção equatorial de cinco frutos foi exposta à solução de iodo-iodeto de potássio (I-KI) e, por meio de análise visual, o índice de degradação do amido foi estimado, utilizando-se escala de 1 a 9 (Bender \&Ebert, 1985), em que 1 e 9 representam mínima e máxima degradação de amido, respectivamente. O conteúdo de sólidos solúveis foi determinado com o uso de refratômetro com compensação automática de temperatura (Atago). A cor de fundo dos frutos foi estimada com auxílio de escala de 1 a 5 , em que 1 e 5 representam frutos com cor de fundo amarelada e esverdeada, respectivamente. A concentração de etileno nos frutos foi determinada conforme descrito por Argenta 
et al. (2006), sendo a avaliação efetuada em 12/02/2007, quatro dias após o ponto de colheita do tratamento testemunha.

A partir dos quatro dias depois de atingido o ponto de colheita no tratamento testemunha, foi iniciada a contagem de frutos caídos e determinada sua proporção, em percentagem, em relação ao número total de frutos em cada planta, obtendo-se a queda de frutos em pré-colheita, aos 4, 14, 27, 35 e 42 dias após o ponto de colheita do tratamento testemunha (DAPC). A colheita dos frutos foi realizada aos 4, 13, 24, 28, 35 e 42 DAPC, com o que, por meio da relação do número de frutos colhidos, por data, com o número total de frutos colhidos por planta, obtevese a percentagem de frutos colhidos por data de colheita. Os frutos colhidos foram contados e pesados, em todas as colheitas efetuadas, e, a partir da relação entre a massa e o número total de frutos colhidos, foi obtida a massa média dos frutos.

$\mathrm{O}$ delineamento experimental utilizado foi em blocos casualizados, com cinco repetições, compostas por uma planta cada. A normalidade e a homogeneidade de variâncias dos dados obtidos para cada variável foram verificadas pelo teste Kolmogorov-Smirnov e pelo teste Bartlett (Sas Institute Inc, 2004), respectivamente. As variáveis queda de frutos em pré-colheita e percentagem de frutos colhidos por data de colheita foram transformadas pela equação $(\mathrm{x}+1)^{0,5}$, a fim de atender às pressuposições da análise de variância. Os resultados foram submetidos à análise de variância, sendo efetuada a comparação de médias pelo teste Tukey, a 5\% de probabilidade de erro, nas variáveis que revelaram significância pelo teste $\mathrm{F}(\mathrm{p}<0,05)$.

\section{RESULTADOS E DISCUSSÃO}

A queda de frutos em pré-colheita foi reduzida pela aplicação de AVG, onde o tratamento testemunha apresentou o maior número de frutos caídos, em todas as épocas de amostragens efetuadas. Não foram observadas diferenças significativas entre os tratamentos com AVG em todas as épocas de amostragem, assim, como na percentagem acumulada de frutos caídos (Figura 1). Ao final do período de avaliação, o tratamento testemunha apresentou $34,9 \%$ de frutos caídos, enquanto os tratamentos com AVG apresentavam de 4,2 a 6,4\% dos frutos caídos em pré-colheita. Essa resposta evidencia a eficiência do AVG no controle da queda pré-colheita de maçãs, permitindo retardar a colheita dos frutos. Todos os tratamentos de AVG apresentaram quedas de frutos pré-colheita insignificantes, até 27 dias após o ponto de colheita, mostrando um período efetivo de controle de 27 a 35 dias. De acordo com Ward et al. (1999), a queda pré-colheita de maçã é decorrente do aumento da síntese e da atividade das enzimas celulase e poligalacturonase, na zona de abscisão dos frutos, estimulados pelo aumento da concentração de etileno nos frutos. Na Figura 2, pode ser constatado que os níveis de etileno em frutos não tratados com AVG são significativamente superiores aos níveis observados nos frutos tratados, independentemente da época e da concentração de AVG utilizada. Como o AVG atua na inibição da biossíntese de etileno (Greene, 2005), a síntese e a atividade das enzimas relacionadas com a abscisão de frutos são diminuídas, justificando os menores índices de queda pré-colheita de frutos, observados nos tratamentos com AVG, em relação ao tratamento controle.

Aos quatro dias após o ponto de colheita, o tratamento testemunha apresentou maior conteúdo de sólidos solúveis em relação aos tratamentos com AVG (Figura 3). Esta resposta pode ser associada à maior hidrólise de amido em açúcares solúveis, no tratamento testemunha, em comparação com a dos demais tratamentos com AVG, pois, segundo Greene (2006), a aplicação de AVG pode reduzir os teores de sólidos solúveis nos frutos, presumivelmente por causa do atraso ou da menor hidrólise de amido. A aplicação de $120 \mathrm{~g} \mathrm{ha}^{-1}$ de AVG, 4 semanas antes do ponto de colheita, proporcionou os menores teores de sólidos solúveis totais, aos 4, 13, 27 e 35 dias após o ponto de colheita; porém, aos 42 DAPC não foram verificadas diferenças significativas entre os tratamentos testados.

Os índices de iodo-amido foram influenciados pelos tratamentos com AVG (Figura 3) e os maiores índices de iodo-amido, aos 4 e 13 DAPC, foram observados no tratamento testemunha. Aos 4 DAPC, a aplicação de $30 \mathrm{~g} \mathrm{ha}^{-1}$ AVG (1 SAPC) + (30 g ha $\left.{ }^{-1}\right)$ AVG (PC) + $30 \mathrm{~g} \mathrm{ha}^{-1}$ AVG (7 DAPC) foi o único tratamento que diferiu, significativamente, dos demais tratamentos com AVG (Figura 3). Aos 27 DAPC, os tratamentos testados não diferiram, significativamente, nos índices de iodo-amido. Nas últimas duas épocas de amostragem (35 e 42 DAPC), a aplicação de 120 $\mathrm{g} \mathrm{ha}^{-1}$ de AVG (4 SAPC) proporcionou a menor degradação de amido em açúcares solúveis, sendo que os demais tratamentos com AVG não diferiram significativamente da testemunha.

O tratamento testemunha apresentou a menor firmeza de polpa $\left(17,5 \mathrm{lb} \mathrm{cm}^{-2}\right)$ aos quatro dias após o ponto de colheita; porém, a aplicação de $120 \mathrm{~g} \mathrm{ha}^{-1}$ de AVG (4 SAPC) e a de $90 \mathrm{~g} \mathrm{ha}^{-1}$ de AVG (2 SAPC) foram os únicos tratamentos que diferiram, significativamente, da testemunha. Aos 13 DAPC, não foram observadas diferenças significativas entre os tratamentos. Na avaliação posterior (27 DAPC), o tratamento testemunha teve maior perda de firmeza de polpa, apresentando $13,4 \mathrm{lb} \mathrm{cm}^{-2}$, enquanto os demais tratamentos com AVG apresentavam, em média, $16,5 \mathrm{lb} \mathrm{cm}^{-2}$. A resposta da firmeza de polpa ao AVG está diretamente relacionada com a redução da síntese de etileno (Steffens et al., 2005), visto que a presença de etileno é necessária para a atividade de enzimas responsáveis pela perda de firmeza 
de polpa (Johnston et al., 2001). Ao final do período avaliado, a menor perda de firmeza de polpa foi observada quando aplicados $120 \mathrm{~g} \mathrm{ha}^{-1}$ de AVG (4 SAPC) e quando aplicados $30 \mathrm{~g} \mathrm{ha}^{-1}$ de AVG $(1 \mathrm{SAPC})+\left(30 \mathrm{~g} \mathrm{ha}^{-1}\right) \operatorname{AVG}(\mathrm{PC})+30 \mathrm{~g}$ $\mathrm{ha}^{-1}$ de AVG (7 DAPC), não existindo diferenças significativas entre esses tratamentos.

As maçãs tratadas com AVG, indiferentemente da época, concentração e parcelamento de aplicações, apresentaram frutos com cor de fundo da epiderme mais verdes do que os do tratamento testemunha, em vista dos menores valores obtidos para esta variável, aos 4 DAPC (Tabela 1). Entre os tratamentos com AVG, a aplicação de $60 \mathrm{~g}$ ha $^{-1}$ de AVG (4 SAPC) + $60 \mathrm{~g} \mathrm{ha}^{-1}$ de AVG (2 SAPC) determi- nou frutos com cor de fundo da epiderme mais esverdeada. Para Steffens et al. (2006), o efeito do AVG sobre a redução do amarelecimento da epiderme deve estar relacionado com a menor atividade das enzimas envolvidas na degradação das clorofilas, em função da menor síntese de etileno. Aos 14 DAPC, o maior grau de amarelecimento da epiderme foi observado nos frutos do tratamento testemunha, diferenciando-se, significativamente, dos tratamentos com AVG (Tabela 1). As diferenças entre tratamentos, quanto à cor de fundo da epiderme, foram minimizadas aos 42 DAPC, sendo a aplicação de $120 \mathrm{~g} \mathrm{ha}^{-1}$ de AVG (4 SAPC) o único tratamento que diferiu, significativamente, da testemunha.

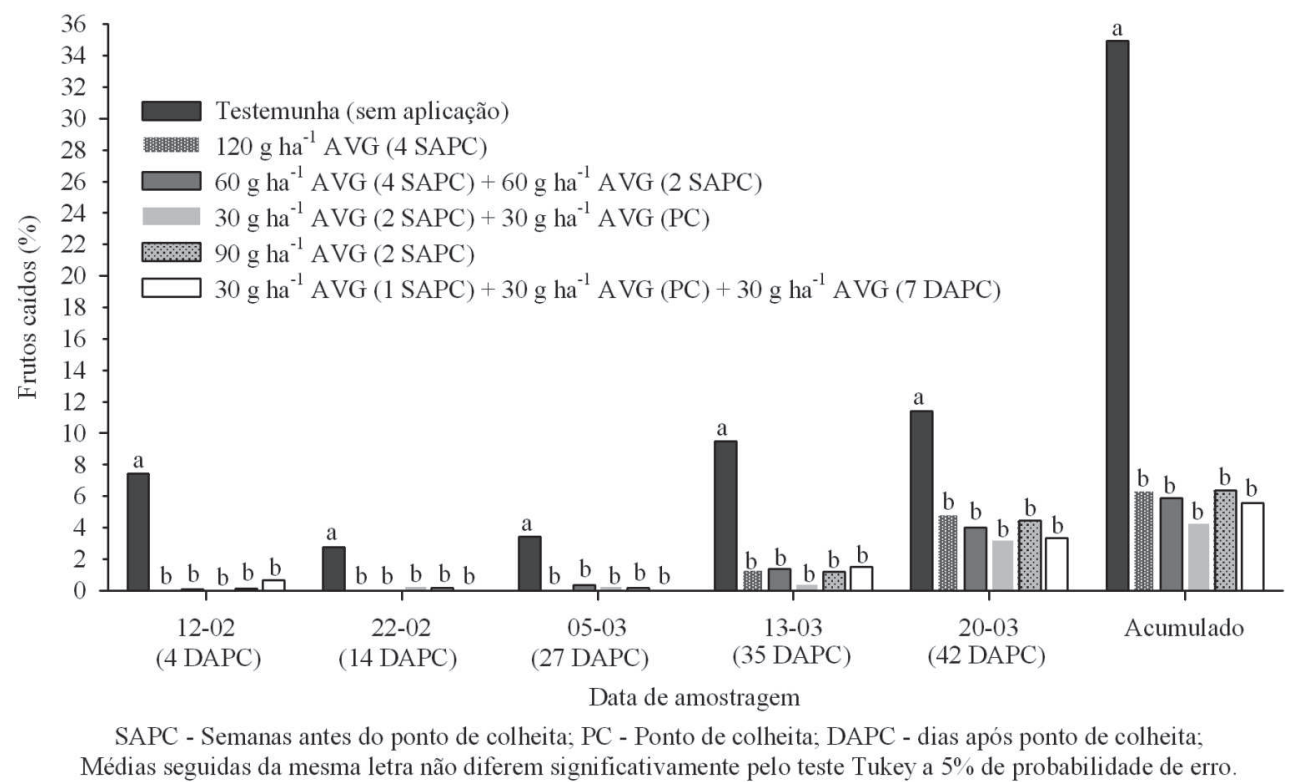

Figura 1. Queda pré-colheita de frutos por época de amostragem e queda pré-colheita acumulada em macieiras 'Imperial Gala', em distintos tratamentos com aminoetoxivinilglicina (AVG).

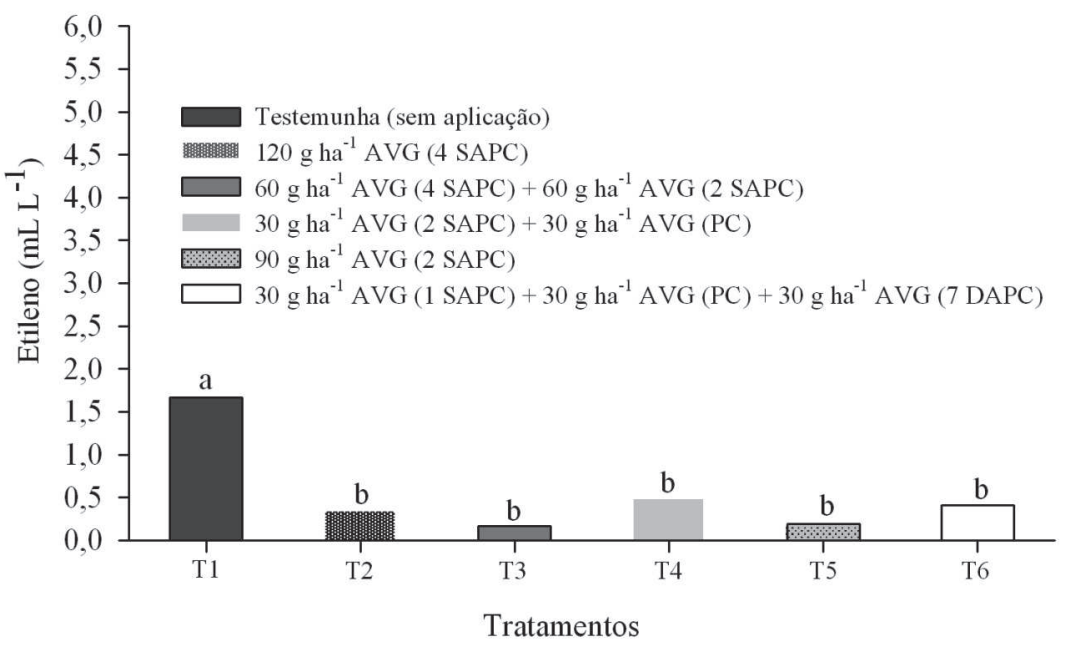

SAPC - Semanas antes do ponto de colheita; PC - Ponto de colheita; DAPC - dias após ponto de colheita; Médias seguidas da mesma letra não diferem significativamente pelo teste Tukey a $5 \%$ de probabilidade de erro.

Figura 2. Níveis de etileno em maçãs 'Imperial Gala', tratadas com aminoetoxivinilglicine (AVG). 


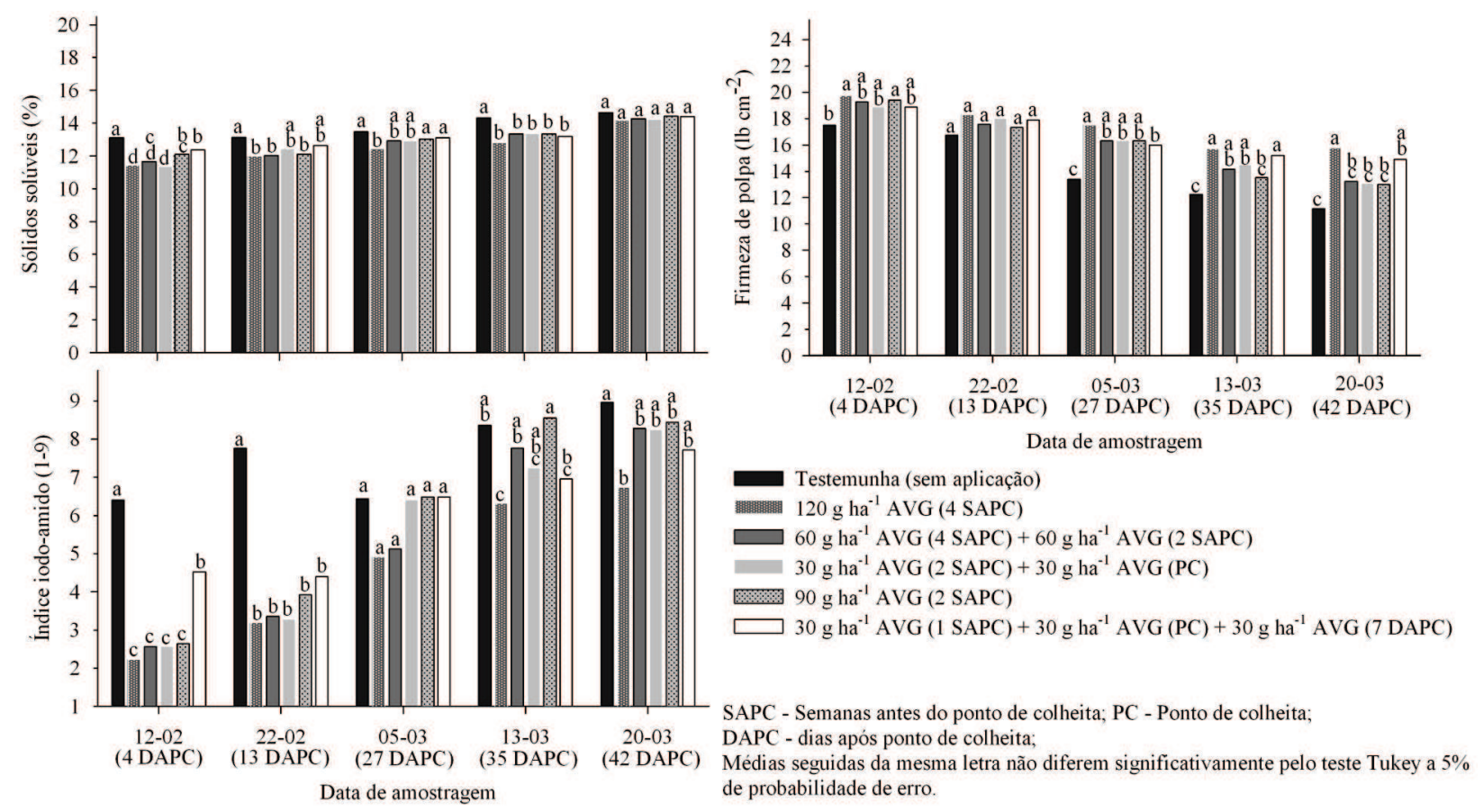

Figura 3. Sólidos solúveis, firmeza de polpa, e índice de iodo-amido, por época de amostragem, em maçãs 'Imperial Gala', em distintos tratamentos com AVG.



SAPC - Semanas antes do ponto de colheita; PC - Ponto de colheita; DAPC - dias após ponto de colheita: Médias seguidas da mesma letra não diferem significativamente pelo teste Tukey a $5 \%$ de probabilidade de erro.

Figura 4. Percentagem de frutos colhidos, por data, em macieiras 'Imperial Gala', em distintos tratamentos com aminoetoxivinilglicine (AVG).

O uso de AVG prorrogou o início da colheita, em relação ao das plantas não tratadas, como também verificado por Amarante et al. (2002), Stover et al. (2003), Phan-Thien et al. (2004), Petri et al. (2006) e Petri et al. (2007). Essa resposta concorda com as obtidas quanto a sólidos solúveis, índice de iodo-amido, firmeza de polpa e cor de fundo dos frutos, que são indicadores do ponto de colheita e que foram influenciadas pela aplicação de AVG. A aplicação de AVG determinou atraso, em 20 dias, na primeira colheita, em relação ao tratamento testemunha (Figura 4). A colheita do tratamento testemunha foi efetuada em três datas, sendo o maior volume colhido aos 20 dias após a primeira colheita, correspondendo a $43,3 \%$ dos frutos. A maior quantidade de frutos colhidos, quando aplicados $30 \mathrm{~g} \mathrm{ha}^{-1} \mathrm{de}$ AVG (1 SAPC) $+30 \mathrm{~g} \mathrm{ha}^{-1}$ de AVG (PC) $+30 \mathrm{~g}$ ha $^{-1}$ de AVG (7 DAPC), foi obtida aos 24 DAPC, sendo efetuada a colheita de $40,6 \%$ dos frutos deste tratamento. Os demais tratamentos com AVG apresentaram a maior proporção de frutos colhida aos 24 dias após a primeira colheita do tratamento testemunha.

As massas médias dos frutos, nos tratamentos com $120 \mathrm{~g} \mathrm{ha}^{-1}$ de AVG, aplicados quatro semanas antes do 


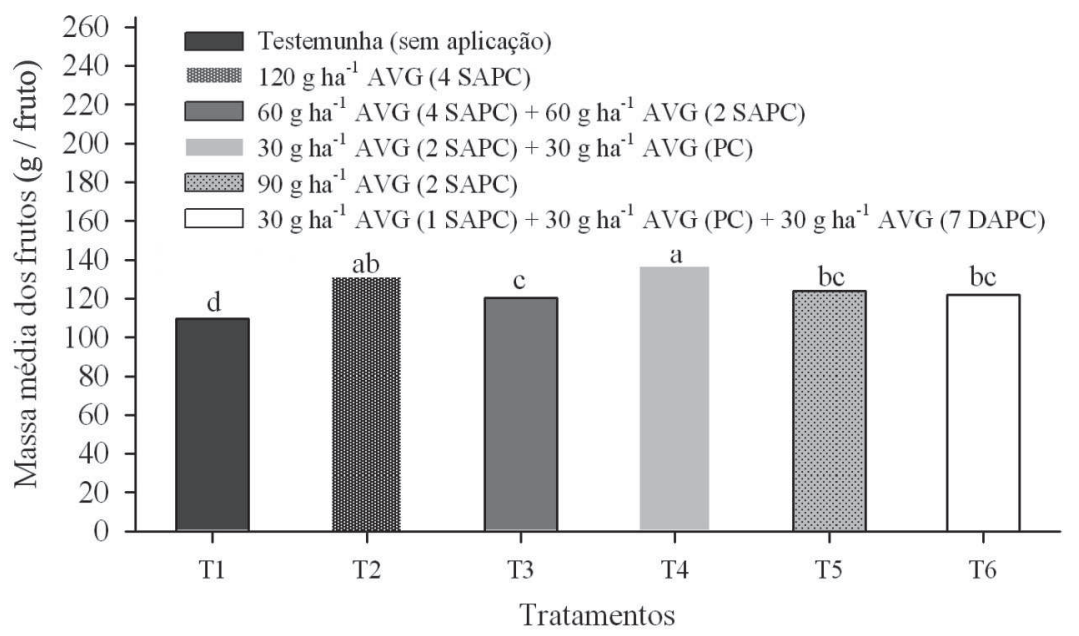

SAPC - Semanas antes do ponto de colheita; PC - Ponto de colheita; DAPC - dias após ponto de colheita; Médias seguidas da mesma letra não diferem significativamente pelo teste Tukey a $5 \%$ de probabilidade de erro.

Figura 5. Massa média dos frutos, em maçãs 'Imperial Gala', tratadas com aminoetoxivinilglicina (AVG).

Tabela 1. Cor de fundo da epiderme de maçãs 'Imperial Gala', em resposta a tratamentos com AVG

\begin{tabular}{lccc}
\hline & \multicolumn{3}{c}{ Cor de fundo $^{\mathbf{a}}$} \\
\cline { 2 - 4 } Tratamento & $\mathbf{1 2 / 0 2}$ & $\mathbf{2 2 / 0 2}$ & $\mathbf{2 0 / 0 3}$ \\
\cline { 2 - 4 } & $4 \mathrm{DAPC}$ & $14 \mathrm{DAPC}$ & $42 \mathrm{DAPC}$ \\
\hline 1) Testemunha & $4,10 \mathrm{a}$ & $4,82 \mathrm{a}$ & $4,94 \mathrm{a}$ \\
2) $120 \mathrm{~g} \mathrm{ha}^{-1} \mathrm{AVG}(4 \mathrm{SAPC})$ & $1,72 \mathrm{bc}$ & $2,60 \mathrm{c}$ & $4,04 \mathrm{~b}$ \\
3) $60 \mathrm{~g} \mathrm{ha}^{-1} \mathrm{AVG}(4 \mathrm{SAPC})+60 \mathrm{~g} \mathrm{ha}^{-1} \mathrm{AVG}(2 \mathrm{SAPC})$ & $1,52 \mathrm{c}$ & $2,42 \mathrm{c}$ & $4,48 \mathrm{ab}$ \\
4) $30 \mathrm{~g} \mathrm{ha}^{-1} \mathrm{AVG}(2 \mathrm{SAPC})+30 \mathrm{~g} \mathrm{ha}^{-1} \mathrm{AVG}(\mathrm{PC})$ & $2,12 \mathrm{bc}$ & $2,86 \mathrm{bc}$ & $4,20 \mathrm{ab}$ \\
5) $45 \mathrm{~g} \mathrm{ha}^{-1} \mathrm{AVG}(4 \mathrm{DAPC})+45 \mathrm{~g} \mathrm{ha}^{-1} \mathrm{AVG}(13 \mathrm{DAPC})$ & $2,74 \mathrm{~b}$ & $3,60 \mathrm{ab}$ & $4,90 \mathrm{ab}$ \\
6) $90 \mathrm{~g}^{-h^{-1}} \mathrm{AVG}(2 \mathrm{SAPC})$ & $2,20 \mathrm{bc}$ & $2,94 \mathrm{bc}$ & $4,44 \mathrm{ab}$ \\
7) $30 \mathrm{~g} \mathrm{ha}^{-1} \mathrm{AVG}(1 \mathrm{SAPC})+30 \mathrm{~g} \mathrm{ha}^{-1} \mathrm{AVG}(\mathrm{PC})+30 \mathrm{~g} \mathrm{ha}{ }^{-1} \mathrm{AVG}(7 \mathrm{DAPC})$ & $2,64 \mathrm{bc}$ & $3,00 \mathrm{bc}$ & $4,48 \mathrm{ab}$ \\
\hline Prob $>\mathrm{F}$ & 0,0001 & 0,0001 & 0,0252 \\
\hline CV $(\%)$ & 24,38 & 12,70 & 9,68 \\
\hline
\end{tabular}


da mesma letra na coluna não diferem significativamente pelo teste Tukey, a $5 \%$ de probabilidade de erro.

ponto de colheita presumido (SAPC), $30 \mathrm{~g} \mathrm{ha}^{-1}$ de AVG (2 SAPC) $+30 \mathrm{~g} \mathrm{ha}^{-1}$ de AVG no ponto de colheita (PC), $90 \mathrm{~g} \mathrm{ha}^{-1}$ de AVG (2 SAPC) e $30 \mathrm{~g} \mathrm{ha}^{-1} \mathrm{de} \mathrm{AVG} \mathrm{(1} \mathrm{SAPC)}$ + $30 \mathrm{~g} \mathrm{ha}^{-1}$ de AVG (PC) + $30 \mathrm{~g} \mathrm{ha}^{-1}$ de AVG (7 DAPC) apresentaram-se superiores à do tratamento testemunha (Figura 5). Greene (2002) e Greene (2006) constatam a possibilidade de aumento dos frutos, em cerca de $1 \%$, a cada dia a mais que permanecem na planta, por atraso no amadurecimento, proporcionado pelo AVG. O aumento da massa média dos frutos pode ser justificado pelo atraso do início da colheita, determinando aumento do período entre a floração e a maturação. A aplicação de $30 \mathrm{~g} \mathrm{ha}^{-1}$ de AVG (2 SAPC) + $30 \mathrm{~g} \mathrm{ha}^{-1} \mathrm{de}$ AVG (PC) + $30 \mathrm{~g} \mathrm{ha}^{-1}$ de AVG (7 DAPC) aumentou em $24,9 \%$ a massa média dos frutos, em relação à do tratamento testemunha. Vários autores constatam o aumento da massa média dos frutos, em resposta a aplicação de AVG, entre os quais podem ser destacados Amarante et al. (2002) que observaram aumento de $12,5 \%$, e Petri et al. (2007) que observaram aumentos variando de 9,1 a $16,3 \%$, na massa média de maçãs 'Gala'. Este aumento da massa média dos frutos pode contribuir expressivamente para o aumento da produtividade, como observado por Venburg et al. (2008), que obtiveram incremento de até $11 \%$ na produtividade de macieiras 'Mondial Gala', decorrente do aumento do calibre dos frutos, proporcionado pelo uso de AVG.

\section{CONCLUSÕES}

O uso de AVG, independentemente da época, concentração e parcelamento das aplicações, reduz a queda précolheita de maçãs 'Imperial Gala'.

A aplicação de $120 \mathrm{~g} \mathrm{ha}^{-1}$ de AVG, quatro semanas antes do ponto de colheita (SAPC), e a aplicação de $30 \mathrm{~g} \mathrm{ha}^{-1}$ de

Rev. Ceres, Viçosa, v. 58, n.5, p. 612-618, set/out, 2011 
AVG (2 SAPC) + $30 \mathrm{~g} \mathrm{ha}^{-1}$ de AVG, no ponto de colheita (PC) $+30 \mathrm{~g} \mathrm{ha}^{-1}$ de AVG, sete dias após ponto de colheita (DAPC), determinaram menor perda de firmeza de polpa de maçãs 'Imperial Gala'.

O grau de amarelecimento da epiderme dos frutos mostra-se menor em frutos tratados com AVG.

O atraso no início da colheita, proporcionado pelo AVG, determinou aumento da massa média dos frutos, cujos melhores resultados foram obtidos com a aplicação de 30 $\mathrm{g} \mathrm{ha}^{-1}$ de $\operatorname{AVG}(2 \mathrm{SAPC})+30 \mathrm{~g} \mathrm{ha}^{-1} \mathrm{de} \operatorname{AVG}(\mathrm{PC})+30 \mathrm{~g}$ ha ${ }^{1}$ de AVG (7 DAPC).

\section{REFERÊNCIAS}

Amarante CVT, Simioni A, Megguer CA \& Blum LEB (2002) Effect of aminoethoxyvinilglicine (AVG) on preharvest fruit drop and maturity of apples. Revista Brasileira Fruticultura, 24:661-664.

Argenta LC (1992) Concentração interna de etileno e maturação de maçãs 'Gala', 'Golden Delicious' e 'Fuji'. Revista Brasileira de Fruticultura, 15:125-132.

Argenta LC, Vieira MJ, Krammes JL, Petri JL \& Basso C (2006) AVG and 1-MCP effects on maturity and quality of apple fruit at harvest and after storage. Acta Horticulturae, 727:495-499.

Bender, RJ \& Ebert, A (1985) Determinação do ponto de colheita de cultivares de macieira. Teste iodo amido. Florianópolis, EMPASC. 6p.

Brackmann A (1992) Produção de etileno, CO2 e aroma de cultivares de maçã. Revista Brasileira de Fruticultura, 14:103-108.

Clayton M, Biasi WV, Southwick SM \& Mitcham EJ (2000) ReTain $^{\mathrm{TM}}$ affects maturity and ripening of 'Bartlett' pear. HortScience, 35:1294-1299.

FAOSTAT. Food and Agriculture Organization of the United Nations Statistical Databases (2010) Disponível em: <http:// f a o s t a t. f a o . o r g / s i t e / 567 / DesktopDefault.aspx?PageID=567\#ancor $>$ Acessado em: 28 de novembro de 2010 .

Greene DW (2002) Preharvest drop control of 'Delicious' apples as affected by aminoethoxyvinylglycine (AVG). Journal of Tree Fruit Production, 3:1-10.

Greene DW (2005) Time of aminoethoxyvinylglycine application influences preharvest drop and fruit quality of 'McIntosh' apples. HortScience, 40:2056-2060.

Greene DW (2006) An update on preharvest drop control of apples with aminoethoxyvinylglycine (ReTain). Acta Horticulturae, 727:311-319.

Johnston JW, Hewett EW, Hertog MLATM \& Harker FR (2001) Temperature induces differential softening responses in apple cultivars. Postharvest Biology and Technology, 23:185-196.
Lurie S (2008) Regulation of ethylene biosynthesis in fruits by aminoethoxyvinylglycine and 1-methylcyclopropene. Acta Horticulturae, 796:31-41.

Petri JL, Leite GB \& Argenta LC (2007) Eficácia do tratamento de AVG no controle da queda e maturação dos frutos de maçã, cultivar Imperial Gala. Revista Brasileira de Fruticultura, 29:239244.

Petri JL, Leite GB, Argenta LC \& Basso C (2006) Ripening delay and fruit drop control in 'Imperial Gala' and 'Suprema' ('Fuji' Sport) apples by applying AVG (Aminoethoxyvinylglycine). Acta Horticulturae, 727:519-525.

Phan-Thien K, Wargo J, Mitchell L, Collett M \& Rath A (2004) Delay in ripening of 'Gala' and 'Pink Lady' apples in commercial orchards following preharvest applications of aminoethoxyvinylglycine. Australian Journal of Experimental Agriculture, 44:807-813.

Sanhueza RMV, Protas JFS \& Freire JM (2006) Manejo da macieira no sistema de produção integrada de frutas. Bento Gonçalves, Embrapa Uva e Vinho. 164p.

Sas Institute Inc (2004) Base SAS ${ }^{\oplus}$ Procedures Guide. Cary, NC SAS Institute Inc. 1861p.

Steffens CA, Amarante CVT, Chechi R, Silveira JPG \& Brackmann A (2008) Aplicação pré-colheita de reguladores vegetais visando retardar a maturação de ameixas 'Laetitia'. Ciência Rural, 39:1369-1373.

Steffens CA, Giehl RFH \& Brackmann A (2005) Maçã 'Gala' armazenada em atmosfera controlada e tratada com aminoetoxivinilglicina e ethephon. Pesquisa Agropecuária Brasileira, 40:837-843.

Steffens CA, Guarienti AFW, Storck L \& Brackmann A (2006) Maturação da maçã 'Gala' com a aplicação pré-colheita de aminoetoxivinilglicina e ethephon. Ciência Rural, 36:434-440.

Stover E, Fargioni MJ, Watkins CB \& Iungerman KA (2003) Harvest management of Marshall 'MacIntosh' Apples: Effect of AVG, ANA, ethephon and summer pruning on preharvest drop and fruit quality. HortScience, 38:1093-1099.

Venburg GD, Hopkins R, Retamales J, Lopez J, Hansen J, Clarke GG, Schröder M \& Rath AC (2008) Recent developments in AVG research. Acta Horticulturae, 796:43-49.

Wang Z \& Dilley DR (2001) Aminoethoxyvinylglycine, combined with ethephon, can enhance red color development without over-ripening apples. HortScience, 36:328-331.

Ward DL, Beers EP, Byers RE \& Marini RP (1999) Cutting apple fruits induces cellulase activity in the abscission zone. HortScience, 34:601-603.

Yuan R \& Carbaugh DH (2007) Effects of ANA, AVG, and 1-MCP on ethylene biosynthesis, preharvest fruit drop, fruit maturity and quality 'Golden Delicious' apples. HortScience, 42:101105 . 